\title{
Article
}

\section{STRAWB2 (Stress and Wellbeing After Childbirth): a randomised controlled trial of targeted self-help materials to prevent post-traumatic stress disorder following childbirth}

Slade, Pauline, West, Helen, Thomson, Gillian, Lane, Steven, Spiby, Helen, Edwards, Rhiannon Tudor, Charles, Joanna M, Garrett, Charlotte, Flanagan, Beverley, Treadwell, Maureen, Hayden, Emma and Weeks, Andrew

Available at http://clok.uclan.ac.uk/31675/

Slade, Pauline, West, Helen, Thomson, Gillian ORCID: 0000-0003-3392-8182, Lane, Steven, Spiby, Helen, Edwards, Rhiannon Tudor, Charles, Joanna M, Garrett, Charlotte, Flanagan, Beverley et al (2020) STRAWB2 (Stress and Wellbeing After Childbirth): a randomised controlled trial of targeted self-help materials to prevent post-traumatic stress disorder following childbirth. BJOG: An International Journal of Obstetrics \& Gynaecology, 127 (7). ISSN 1470-0328

It is advisable to refer to the publisher's version if you intend to cite from the work. http://dx.doi.org/10.1111/1471-0528.16163

For more information about UCLan's research in this area go to http://www.uclan.ac.uk/researchgroups/ and search for <name of research Group>.

For information about Research generally at UCLan please go to http://www.uclan.ac.uk/research/

All outputs in CLoK are protected by Intellectual Property Rights law, including Copyright law. Copyright, IPR and Moral Rights for the works on this site are retained by the individual authors and/or other copyright owners. Terms and conditions for use of this material are defined in the policies page. 
Article type : Randomised controlled trial

STRAWB2 (Stress and Wellbeing After Childbirth): a randomised controlled trial of targeted self-help materials to prevent post-traumatic stress disorder (PTSD) following childbirth

Professor Pauline Slade (Corresponding author)

Ground Floor Whelan Building

Department of Psychological Sciences

University of Liverpool,

Liverpool

L69 3GB

Pauline.slade@liverpool.ac.uk tel 01517945485

Dr Helen West

Institute of Health and Life Sciences

University of Liverpool

Liverpool

L69 3GB

\section{Dr Gill Thomson}

This article has been accepted for publication and undergone full peer review but has not been through the copyediting, typesetting, pagination and proofreading process, which may lead to differences between this version and the Version of Record. Please cite this article as doi: $\underline{10.1111 / 1471-0528.16163}$

This article is protected by copyright. All rights reserved 
School of Community Health and Midwifery

Brook Building, BB243

University of Central Lancashire

Preston

Lancs

Dr Steven Lane

Centre for Medical Statistics and Health Evaluation

University of Liverpool,

Liverpool

Professor Helen Spiby

School of Health Sciences

University of Nottingham

Nottingham

NG7 2RD

Professor Rhiannon Tudor Edwards

Centre for Health Economics and Medicines Evaluation

Bangor University

Gwynedd

Dr Joanna M Charles

This article is protected by copyright. All rights reserved 
Centre for Health Economics and Medicines Evaluation

Bangor University

Gwynedd

Dr Charlotte Garrett

Lancashire Teaching Hospitals NHS Foundation Trust

Preston

Lancs

Ms Beverley Flanagan

Lancashire Teaching Hospitals NHS Foundation Trust

Preston

Lancs

Ms Maureen Treadwell

Birth Trauma Association

Mrs Emma Hayden

Expert by Experience

Liverpool Womens Hospital Foundation Trust

Liverpool

Professor Andrew Weeks

This article is protected by copyright. All rights reserved 
Department of Women's and Children's Health

University of Liverpool

Liverpool Womens Hospital Foundation Trust and Liverpool Health Partners

Liverpool

Shortened running title: Preventing Post Traumatic Stress after Childbirth

This article is protected by copyright. All rights reserved 


\begin{abstract}
:
Background

Post-traumatic stress disorder (PTSD) can develop after a traumatic childbirth.
\end{abstract}

\title{
Objective
}

To test if providing psychological self-help materials would significantly lower the incidence of PTSD at 6-12 weeks postnatally.

\section{Design}

Open label, randomised controlled trial, blinded outcome assessment.

\section{Setting}

Community midwifery services in two North West NHS Trusts

\section{Sample}

2419 women receiving usual NHS postnatal care

\section{Methods}

Midwives screened women for traumatic birth experience. 678 women who screened positive $(28.1 \%)$ were randomly allocated to self-help with usual care $(n=336)$ or usual care alone $(n=342)$. Self-help materials, were a leaflet and on-line film designed to prevent the development of PTSD after trauma exposure through how to manage early psychological responses.

\section{Main Outcome Measure}

The primary outcome was a composite of diagnostic and sub-diagnostic PTSD at 6-12 weeks postnatally using the gold standard Clinician Administered PTSD Interview (CAPS-5).

\section{Results}

478 of $678(70.5 \%)$ correctly randomised women and 9 randomised in error were followed up. Diagnostic or sub-diagnostic PTSD rates at follow-up did not differ between groups who received self-help $(26.7 \%, 65 / 243)$ or usual care alone $(26.2 \%, 64 / 244)$ (ITT analysis: relative risk (RR) $1.02,95 \%$ confidence interval (CI) 0.68 to 1.53 ). Findings remained consistent in the per protocol 
analysis (RR 1.04, 95\% CI 0.85 to 1.27 ). Women viewed the materials very positively. There were no adverse effects. Health economic micro-costing indicated implementation would be very low cost.

\section{Conclusions}

Many women experience a traumatic birth and risk developing PTSD, but self-help strategies without professional support are insufficient and should not be routinely introduced.

\section{Funding}

NIHR:Research for Patient Benefit Programme (Grant:PB-PG 021536037) awarded after external peer review

Key Words: Post traumatic stress disorder, postnatal, childbirth, prevention, randomised controlled trial

\section{Trial registration ISRCTN 44832384}

Tweetable abstract. Self-help information alone does not reduce the number of women developing PTSD after a traumatic childbirth. 


\section{Introduction}

Post-traumatic stress disorder (PTSD) after childbirth is a major cause of psychological distress, with 3\% of women suffering at full diagnostic levels and 5-9\% when sub-diagnostic levels (partial PTSD) are included ${ }^{1}$. When childbirth is experienced as traumatic, defined as high fear of death or damage to self or baby during or shortly after childbirth, then women are at risk of developing PTSD $^{2-5}$. Other potential risk factors for PTSD include poor quality of interactions with staff, medical interventions and previous psychiatric history or trauma ${ }^{6}$. PTSD is debilitating and in the absence of intervention tends to become chronic. As well as the distress for the woman PTSD can adversely impact the child's cognitive, emotional and social development ${ }^{7,8}$ Prevention where possible is therefore crucial.

Experiencing an event as traumatic does not inevitably lead to PTSD. Intrusive experiences involving imageryand thoughts are normal responses to trauma that facilitate memory processing. Where women view these as signs of illness or not coping and avoid these responses this contributes to traumatic memories remaining unprocessed leading to PTSD9.

The STRAWB2 self-help materials (a leaflet and film) were designed to prevent the development of PTSD. Experts by experience guided development to ensure accessibility, and materials were piloted in a feasibility study ${ }^{10}$. The materials derive from evidence-based psychological theory ${ }^{11}$. They incorporate explanations

1) Why women experience distressing responses to help normalize these responses and reduce their negative evaluation.

2) Why it is important not to block unpleasant images and thoughts.

3) How supportive discussions help memory processing and provides an exercise to identify a suitable person, time and place with whom to do this.

4) Exercises using implementation intentions throughout to help women translate their new understandings into actions.

PTSD, treatment is expensive, so a simple and low-cost prevention package is attractive. However, the evidence on whether psychoeducation and self-help can prevent PTSD is limited and inconsistent ${ }^{12,13}$. NICE antenatal and postnatal mental health guidelines ${ }^{14}$ recommend researchers 
develop effective psychological interventions for perinatal women, including gathering evidence of cost-effectiveness..

This study aimed to evaluate whether providing self-help materials to women who have experienced a traumatic childbirth reduced the incidence of PTSD 6-12 weeks postnatally at diagnostic and subdiagnostic levels when compared with usual care and to provide a health economic analysis. PTSD symptoms postnatally are particularly important because of transitions in and the formation of new relationships. As a result of this critical salience NICE recommends that intervention is not restricted to those with diagnostic level symptoms and indeed childbirth trauma services intervene at non diagnostic levels. Therefore the protocol prespecified this combined outcome as the most clinically appropriate. The specific criteria were chosen on the basis of the existing literature. This helpfully meshed with the targeted dimensions of the prevention information. 


\section{Methods}

\section{Study design}

STRAWB2 (Stress and Wellbeing After Childbirth) was a phase III multi-site randomised controlled trial(RCT), evaluating whether providing a targeted sample of women with self-help materials reduced the incidence of PTSD after childbirth when compared with usual care. We included clinical and economic evaluation of cost per case prevented, and qualitative feedback from women on the self-help materials. 125 community midwives were trained to recruit and randomise women, in accordance with Good Clinical Practice standards.

\section{Participants}

Women aged 16 years or over, who had given birth to a live baby, and had sufficient English language to complete the measures were eligible. An xxclusion criterion wasthose receiving other specialist services (enhanced midwifery for drug/alcohol or social care reasons, or perinatal mental health teams). Study sites were Liverpool Women's NHS Foundation Trust and Lancashire Teaching Hospitals NHS Foundation Trust with recruitment from May 2017 to September 2018.

\section{Randomisation and masking}

Eligible women were informed about the study at their first postnatal visit (home or community). At a subsequent routine contact, following completion of routine postnatal care, and any actions or advice based on clinical judgement, midwives asked women about participation. After providing written informed consent, women were asked the screening questions to identify those who had experienced birth as traumatic. This screening tool was based on DSM-IVR criteria and developed in liaison with the Birth Trauma Association and piloted in the STRAWB feasibility study ${ }^{10}$. Thinking about your childbirth (and any time in hospital after) was there any time during this when you felt (i) horror or helplessness about what was happening? (yes/no) (ii) really frightened about your own or your baby's wellbeing? (yes/no).

This tool incorporates both the perceived threat and the response, as women's appraisal during the birth process is a key risk factor for PTSD onset ${ }^{2,15}$. Women answering 'yes' to either question were randomised to self-help or usual care by their midwife, using an independent web-based system (sealedenvelope.com). Owing to the nature of the materials being tested it was impossible to mask women or midwives from treatment allocation. 


\section{Procedures}

Women allocated to self-help received the leaflet and web-link from the midwife, and a reminder text message two weeks later from a researcher not involved in analysis. All trial participants received routine care from health visitor and GP over the follow up period. Information on demographics, childbirth, and maternal and infant morbidity from women and their hospital records was collected

Women were followed-up by telephone at 6-12 weeks postnatally, at least 4 weeks after randomisation. They completed the CAPS clinical interview with researchers blinded to group allocation and trained to prespecified criterion for reliable rating. Where diagnostic or subdiagnostic PTSD was identified, the woman's health visitor was informed.

\section{Outcomes}

The primary outcome was a composite of diagnostic and sub-diagnostic PTSD, assessed at 6-12 weeks postnatally using the gold standard CAPS-5 clinical interview. This derives directly from the DSM-5 definition of diagnostic PTSD.. Sub-diagnostic PTSD was defined as meeting the diagnostic threshold for criteria A (exposure) and G (distress or impairment in relation to the event), and meeting the diagnostic threshold for at least one symptom from either criteria $\mathrm{B}$ (reexperiencing) or C (avoidance). Secondary outcomes were depression and anxiety Hospital Anxiety and Depression Scale (HADS) ${ }^{16}$, attachment Multidimensional Parental Attachment Scale (MPAS) ${ }^{17}$ ), couple relationship quality (Dyadic Adjustment Scale (DAS4) ${ }^{18}$. Health service use was measured using a bespoke Client Service Receipt Inventory (CSRI) questionnaire reporting all contacts with NHS healthcare professionals from randomisation to follow-up, including consultations relating to birth experience, whether routine or specially organised.

\section{Health economic micro-costing and service use analysis}

Micro-costing was used to detail costs of intervention delivery ${ }^{19}$. The intervention developers (PS, HW) provided information regarding the cost of the self-help materials (leaflet and film), training and number of midwives trained in the trial to deliver the intervention. Midwives were surveyed to identify time taken for screening and information provision to screen positive women. 
The micro-costing and a cost-consequence analysis were conducted from a service provider (NHS) perspective using national unit costs for 2016-17) ${ }^{20,21}$.

\section{Feedback interviews}

To assess use of the leaflet and film, a convenience subsample of women in the self-help arm completed a telephone interview covering:

(i) Whether they had used the materials;

(ii) What had been helpful or unhelpful;

(iii) Any actions taken as a result of the prevention information.

Descriptive (frequencies) and thematic analysis ${ }^{22}$ of the responses was undertaken.

\section{Sample size and statistical analysis}

Considering only screen positive women, to detect a reduction of PTSD cases from $25 \%$ to $15 \%$ at 6-12 weeks follow up required a sample size of 247 women in each group ( $80 \%$ power at $5 \%$ significance level). We analysed the primary outcome for both intention-to-treat (ITT) and per protocol levels. For the latter, women who had screened negative to traumatic birth but were randomised in error were excluded. The baseline demographic and clinical data were summarised using standard summary statistics. For all primary and secondary outcomes relative risks or mean differences, with $95 \%$ confidence intervals are reported.

Standard hypothesis tests, chi-squared, independent sample t-test etc. were used to determine if there were any between-group differences in the primary and secondary outcome measures. Logistic regression analysis was also used to calculate adjusted odds ratios for the primary outcomes when controlling for the influence of known confounding variables. All hypothesis testing was undertaken at the 5\% significance level.

\section{Patient and Public Involvement}

Patients and public representatives were integral members of the trial management group and their invaluable insights influenced the study from its inception, through implementation interpretation, and dissemination. Our strategy incorporated national and local perspectives via the Birth Trauma Association charity and a local expert by experience. 


\section{Funding}

This research trial was funded by the National Institute for Health Research (NIHR) under its Research for Patient Benefit (RfPB) Programme (Grant Reference Number PB-PG 021536037).

\section{Results}

Community midwives invited 3444 eligible women to participate. Of these, 2414 women consented and were asked the two screening questions. 678 women screened positive $(28.1 \%)$ and were randomly allocated to either self-help with usual care $(n=336)$ or usual care alone $(n=342)$. These women were included in the intention to treat and per protocol analyses. An additional 40 women who had screened negative were randomised in error to self-help with usual care $(n=25)$ or usual care alone $(\mathrm{n}=15)$, were included in the intention to treat analysis. Any additional randomisation violations and how managed are shown in Fig1.

\section{Site comparisons}

355 women were randomised at Liverpool Women's NHS Foundation Trust, and 363 at Lancashire Teaching Hospitals NHS Foundation Trust (Preston and Chorley). The sites differed only in the number of days postnatal when randomisation took place (median of 24 Liverpool and 12 Lancashire) reflecting differences in midwifery services. A greater proportion of women in Liverpool lived in areas of higher deprivation. The demographic, obstetric and infant data of the 678 randomised women were similar in the two trial sites (Table S1).

\section{The sample in context}

Compared with all women who gave birth at these two locations during the study period, women who screened positive were more likely to have: induction of labour, birth in theatre, instrumental birth, emergency Caesarean section, blood loss over 1000ml, and infant Apgar $<7$ at 5 minutes. A higher proportion of White British women took part, likely due partly to the inclusion criterion of sufficient English language (Table S2).

\section{Baseline comparisons for self-help and usual care groups}

Baseline characteristics were comparable between the groups, except for induction of labour: selfhelp $53.2 \%$ (183/344) and usual care 43.3\% (146/337). There was a trend towards more women in the self-help group having had skin-to-skin contact with their baby following birth: self-help 
$77.3 \%$ (265/313), usual care 72.2\% (242/306) and having experienced blood loss over 1000ml: self-help 19.7\% (68/344), usual care 15.1\% (51/337). More women who had assisted conception were randomised to usual care $(4.1 \%, 14 / 338)$ than self-help $(0.9 \%, 3 / 342)$, although numbers are small (Table 1).

\section{Follow-up}

We successfully followed-up 478 women who had been correctly randomised to self-help or usual care (70.7\%) at 6-12 weeks postnatally and at least 4 weeks after randomisation, and an additional 9 women who had been randomised in error (Figure 1).

\section{Primary outcome}

Using an intention to treat (ITT) analysis the proportion of women with diagnostic or subdiagnostic PTSD at follow-up did not differ between groups who received self-help materials $(26.7 \%, 65 / 243)$ or usual care alone $(26.2 \%, 64 / 244)$ (relative risk (RR) 1.02, 95\% confidence interval (CI) 0.68 to $1.53, \mathrm{P}=0.92$ ) (Table 2). Findings remained consistent in the per protocol analysis, excluding a small number of screen negative women randomised by midwives in error (RR 1.04, 95\% CI 0.85 to 1.27 (table S3), and when the ITT analysis was adjusted for induction and blood loss over 1000ml: (adjusted odds ratio (AOR) 0.99, 95\% CI 0.65 to 1.49 ) (Table 3).

\section{Secondary outcomes}

There were no differences identified in the ITT analysis of secondary outcomes of usual care alone versus with self-help at follow-up, including whether women met the symptom threshold for criterion A: exposure to a traumatic experience (RR 0.99, 95\% CI 0.70 to 1.39 ), criterion B: intrusion symptoms (RR $0.94,95 \%$ CI 0.78 to 1.12 ), criterion C: avoidance symptoms (RR 0.85 , 95\% CI 0.70 to 1.04 ), criterion D: cognitions and mood symptoms (RR $0.98,95 \%$ CI 0.80 to 1.19), criterion E: arousal and reactivity symptoms (RR $0.93,95 \%$ CI 0.73 to 1.17 ), criterion G: distress or impairment (RR 1.01, 95\% CI 0.83 to 1.22) (Table 2).

The self-help materials were particularly targeted at symptoms in criteria B and C, and it is worth noting that fewer women in the self-help group experienced these symptoms (criterion B: selfhelp: 87 (37.7\%), usual care: 97 (40.8\%); criterion C: self-help: 46 (19.5\%), usual care: 61 $(25.3 \%))$. However, these differences did not reach statistical significance. 
There were also no differences between women in the self-help versus usual care groups for: anxiety (mean difference (MD) $-0.29,95 \%$ CI -1.03 to 0.45 ), depression (MD $0.31,95 \%$ CI -0.30 to 0.91 ) as measured by the Hospital Anxiety and Depression Scale (HADS) at follow-up; The Multidimensional Parental Attachment Scale(MPAS) questionnaire Quality of attachment to the infant (MD $-0.43,95 \%$ CI -1.30 to 0.50 ), Absence of hostility towards the infant (MD $-0.29,95 \%$ CI -0.93 to 0.35), and Pleasure in interaction with the infant (MD 0.07, 95\% CI -0.57 to 0.72), or the DAS4 questionnaire covering the quality of the couple's relationship (MD -0.04, 95\% CI -0.69 to 0.61 ) (Table 2).

\section{Comparison of screen positive and screen negative women.}

Comparison of the women who screened positive for a traumatic birth $(n=688)$ and those who screened negative $(n=1726)$ showed that those who screened positive were more likely to be nulliparous, but for other demographics the groups were comparable (Table S4).

\section{Comparison of those completing both time points and those lost to the study}

Follow-up was completed for 478 of the 678 women randomised (70.5\%). Comparison of the demographic, obstetric and infant variables between those completed and who did not complete follow-up showed no differences between the groups (Table S5). Of the women followed up, 236 had been randomised to self-help, and 242 to usual care. There were no differences between selfhelp and usual care in women followed-up, apart from those already observed between the groups of women randomised (fewer women in the self-help group had assisted conception, more women in the self-help group had induction of labour, skin-to-skin contact, and blood loss over $1000 \mathrm{ml}$ (Table S5)).

\section{Film analytics}

Film analytics indicated that the film which was hidden from search engines was watched 67 times (to $26^{\text {th }}$ Sept 2018). It was impossible to know if these were different or the same individuals.

\section{CAPS fidelity monitoring}

To ensure consistency between the four researchers conducting CAPS interviews, the transcripts of 143 interviews were coded by two researchers independently: all diagnostic, sub-diagnostic and $20 \%$ of non-diagnostic interviews, until July 2018 . The overall agreement on diagnostic category 
between coders across all interviews was 90.4\%. Cohen's Kappa across all raters for all interviews was 0.80 , classified as excellent ${ }^{23}$.

\section{Feedback interviews}

A convenience sample of 83 (34.4\% of the 241 women randomised to self-help who completed follow-up) took part in a feedback interview. Comparisons of demographic, obstetric and followup data showed no systematic differences between these women and others randomised to selfhelp. Most women remembered receiving the leaflet $(\mathrm{N}=77 / 83 ; 92.8 \%)$ and had read the leaflet $(\mathrm{N}=68 / 75 ; 90.7 \%)$. Of those who had read it, most women read it once $(\mathrm{N}=47 / 70 ; 67.1 \%)$. The majority of women "Agree" $(\mathrm{N}=43 / 69 ; 62.3 \%)$ or "Strongly Agree" $(\mathrm{N}=14 / 69 ; 20.3 \%)$ that they found the leaflet useful. The majority of women "Agree" $(\mathrm{N}=40 / 69 ; 58.0 \%)$ or "Strongly Agree" $(28 / 69 ; 40.6 \%)$ that they found the leaflet easy to understand. Most women did not remember receiving the web-link $(\mathrm{N}=44 / 78 ; 56.4 \%)$ and had not watched the film $(\mathrm{N}=48 / 52 ; 92.3 \%)$. From this sample, only 4 women said they had watched it. Most women preferred a leaflet format $(\mathrm{N}=$ $54 / 68 ; 79.4 \%)$.

The key qualitative findings were:

- Many women liked the design of the materials and information included.

- It helped women understand and to normalise some of the feelings they experienced after birth.

- It helped open channels of communication (including professional and personal support).

- Some suggested that they would like a clearer link to web materials (despite the link being cited twice in the leaflet and embedded in the reminder text message).

- Some suggested the intervention may have been more beneficial if supported by healthcare professionals.

\section{Health economic micro-costing and service use analysis}

Intervention costs within the research context ranged from $£ 4$ to $£ 6$ per woman, based on 2,409 women screened. .

For implementation in a maternity service of 60 midwives, costs would be $£ 3,402$ ( $£ 57$ per midwife) for the set-up year, reducing to $£ 1,731$ ( $£ 29$ per midwife) for subsequent years training 
for returners/new starters and updating. . Using current predominant models of working (noncontinuity) estimates of a case load of 100 women per annum per midwife prorated to 70 to account for part-time working equates to costs of $£ 0.81$ per woman in year of service set up for training of midwives and $£ 0.41$ in maintenance yearsThere is also the cost of the self-help materials ( $£ 0.56$ per screen positive woman prorated to $£ 0.16$ across the postnatal population) and time for the midwives to screen ( 2.8 minutes) and provide materials ( 3 minutes) for those screen positive.. and -

Non-routine service use for both groups was minimal.

\section{Discussion}

\section{Main findings}

We evaluated the effect of providing information about the normality of early trauma responses and how best to manage these for women who had a traumatic birth. This was ineffective in reducing the incidence of PTSD at diagnostic (full) and sub-diagnostic (partial) levels at 6-12 weeks postnatally. Given that there was no difference in the incidence of PTSD, the lack of difference in secondary outcomes was unsurprising. A reduction of PTSD symptoms would have formed the mechanism behind other predicted differences.

Women valued the information, there were no adverse effects, and it did not increase distress. Midwives found it easy to implement the screening tool and administer materials, and it is very low-cost. In its current form, it was insufficient to prevent the development of PTSD following childbirth. Qualitative results indicate that it might be more effective if supported with active input from midwives or health visitors which could facilitate use by giving permission for self-care and through providing practice of the strategies.

\section{Strengths and limitations}

This is the first trial of a self-help intervention derived directly from psychological theory to prevent PTSD following traumatic childbirth. Bias was minimised by using an independent webbased service to generate the randomisation list and conceal allocation. Researchers who assessed outcomes were blinded to allocation, and the inter-rater reliability was high. Samples were well matched and sufficient for power. Follow up rates are acceptable at a typical level for 
psychological intervention studies, and there is no evidence that samples differed on this basis. Clearly those lost to follow up could impact on findings. All outcomes are reported according to the prespecified data management plan, and there is minimal missing data. We believe this trial provides robust evidence.

It is unusual to have 125 community midwives across two sites recruiting to a trial. Overall this worked successfully and enabled ambitious randomisation targets to be reached. The trial design also benefitted from being fitted into usual care to reflect a real world evaluation. The challenges included maintaining consistency and a higher number of women than expected were randomised in error. However, the per protocol analysis shows consistent findings.

Limitations are that the study tested provision rather than use of the self-help materials. The feedback interviews were from a convenience rather than random sample. They indicate that most women read the leaflet but did not access the film. In the first few months with a newborn baby a woman's attention is naturally focused on her infant, and it may be difficult to legitimize or find time to attend to her own self-care. Therefore, women may have found it difficult to prioritise the exercises in the leaflet. Feedback interviews suggested that it may be more effective if midwives or health visitors supported and prompted use of the self-help materials. Due to the study design, we had specifically emphasised in training that midwives should not change their practice, to ensure that women received their usual care before trial procedures were initiated and to avoid exposing women in the usual care group to principles from the self-help materials.

It is possible that the screening triggered women in the control group to access other web based material but the frequency of this was equivalent in both groups $(\mathrm{N}=17)$. In addition the sites women reported using do not have equivalent material to this novel intervention. The model of screening and provision of information tests the broad utility of this package and readiness to utilize and therefore potential effectiveness may be higher in women who actively seeking information. Finally outcomes were only assessed between 6- 12 weeks and PTSD with deferred onset can occur. Longer term follow up might yield different results.

\section{Interpretation}


Leaflets are often introduced into practice without evidence of impact. During the trial we repeatedly encountered attitudes that testing the materials was unnecessary, as a prevention package based on sound psychological principles must be a "good thing". Wessley et al ${ }^{12}$ found that despite the ubiquity of psychoeducation following trauma, evidence supporting its use was rare. Only one direct trial of psychoeducation was identified ${ }^{24}$; an RCT of self-help material for civilian trauma victims presenting at an Accident and Emergency department. There was no evidence of positive impact but the material provided was long, dense and inaccessible.

Indirect evidence concerning the effectiveness of psychoeducation is mixed ${ }^{12}$. Participants receiving psychoeducation in RCTs have had modest improvements, although the interventions were to treat rather than prevent PTSD, and effects may be due to trial participation rather than the

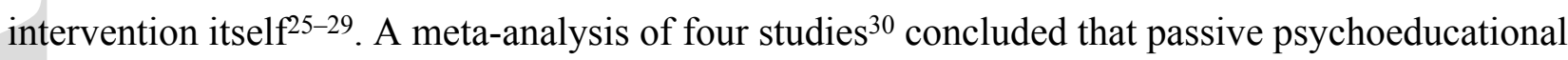
interventions could effectively reduce symptoms of depression and psychological distress.

However, this overall effect masks the finding that there was no improvement in the one included study of psychological distress alone ${ }^{31}$. STRAWB2 materials moved beyond passive psychoeducation: tasks encouraged women to practice adaptive responses to facilitate memory processing, so the studies are not directly comparable. None of these trials focused in the early postnatal period when it may be difficult legitimizing time for self-care and self-help.

A recent systematic review of interventions to prevent PTSD following childbirth(34), concluded that there was insufficient evidence that interventions tested to date prevent PTSD following traumatic childbirth. This study further extends that finding.

\section{Conclusions}

Over a quarter of women in this UK sample experienced birth as traumatic, and $26 \%$ of these women developed diagnostic or subdiagnostic PTSD by 6-12 weeks postnatally. This indicates an overall sample rate of $7.5 \%$ which concurs with existing information [1] and further underlines PTSD after childbirth as a significant problem. A robust test of providing of self-help materials well-grounded in psychological theory, showed these did not prevent the development of PTSD. Although providing information may be considered important, it was inadequate to generate clinical change. Our study should urge caution in the distribution of psychoeducational self-help following trauma, as such minimalist approaches appear to be an ineffective use of resources and 
may provide inappropriate reassurance that a vulnerable group are receiving an appropriate help. When trying to extract maximum value from limited budgets and where the need to be seen to be 'doing something' is powerful, such minimalist approaches whilst superficially attractive, may be false economy in relation to trauma.

\section{Funding and Disclaimer}

This research trial was funded by the National Institute for Health Research (NIHR) under its Research for Patient Benefit (RfPB) Programme (Grant Reference Number PB-PG 021536037). The views expressed are those of the authors and not necessarily those of the NHS, the NIHR or the Department of Health.

\section{Ethical approval}

This was given by the North West - Liverpool Central Research Ethics Committee (16/NW/0680) on $13^{\text {th }}$ December 2016, the Sponsor was the University of Liverpool (UoL001229), and the study was funded by NIHR RfPB (PB-PG-0215-36037).

\section{Transparency statement}

Professor Slade as lead author affirms this manuscript is an honest, accurate and transparent account of the study and no important aspects are omitted. All authors had access to the data and the study was entirely independent from funders.

\section{Author Contributions}

PS was principal investigator and took overall responsibility for the trial. She was instrumental in its conception, planning, carrying out, analysing and writing up. HW was the principal researcher for the trial managing all other researchers and completing data collection and providing first draft of the paper. GT was a site lead and contributed to ensuring quality standards of the work carried out and the interpretation and paper. SL the trial statistician was involved in the conception, design and completed the analysis and contributed to the paper. HS was instrumental in conception, design, provided midwifery oversight and input to the write up. RTE oversaw the health economic aspect was involved in conception, design, analysis and write up. JMC contributed to the health economic design and analysis and contributed to the paper. CG was involved in midwifery training, data collection and oversaw fidelity checking for CAPS5and contributed to the paper. BF 
provided midwifery training and support, data collection and contributed to the paper. MT and EH provided our public and patient involvement. MT on behalf of the Birth Trauma Association advised on all aspects from conception to completion including the paper. EH was involved in advising on the running of the trial, its interpretation, played major role in dissemination activities and contributed to the paper. AW was instrumental in conception, design, provided obstetric oversight and input to the write up.

\section{Conflict of Interest Declaration}

No author has conflicts of interest in relation to the paper. HW's post at University of Liverpool was funded by the NIHR grant to work as a postdoctoral research associate on the trial. All authors declare: no support from or any financial relationships with any organisations that might have an interest in the submitted work in the previous three years and no other relationships or activities that could appear to have influenced the submitted work. Completed disclosure of interest forms are available to view online as supporting information.

\section{Acknowledgements}

We would like to acknowledge our many collaborators: Katie Balling, Jenny Butters, Lucy Bryning Rebecca Crookall , Joanne Goss, Claire Lyon, Katrina Rigby, Afra Qassim, Bernice Shaffri, Sim Steen, Avril Swan, all our midwife recruiters and all the women who participated without whom this work could not have been completed.

\section{References}

1. Yildiz PD, Ayers S, Phillips L. The prevalence of posttraumatic stress disorder in pregnancy and after birth: A systematic review and meta-analysis. J Affect Disord. 2017 Jan 15;208:634-45.

2. Andersen LB, Melvaer LB, Videbech P, Lamont RF, Joergensen JS. Risk factors for developing post-traumatic stress disorder following childbirth: a systematic review. Acta Obstet Gynecol Scand. 2012;91(11):1261-72. 
3. Alcorn KL, O’Donovan A, Patrick JC, Creedy D, Devilly GJ. A prospective longitudinal study of the prevalence of post-traumatic stress disorder resulting from childbirth events. Psychol Med. 2010 Nov 11;40(11):1849-59.

4. Dekel S, Stuebe C, Dishy G. Childbirth induced posttraumatic stress syndrome: A systematic review of prevalence and risk factors. Frontiers in Psychology. 2017.

5. Leeds L, Hargreaves I. The psychological consequences of childbirth. J Reprod Infant Psychol. 2008;26(2):108-22.

6. Grekin R, O’Hara MW. Prevalence and risk factors of postpartum posttraumatic stress disorder: A meta-analysis. Clin Psychol Rev. 2014;34(5):389-401.

7. Garthus-Niegel S, Ayers S, Martini J, Von Soest T, Eberhard-Gran M. The impact of postpartum post-traumatic stress disorder symptoms on child development: A populationbased, 2-year follow-up study. Psychol Med. 2017;

8. Cook N, Ayers S, Horsch A. Maternal posttraumatic stress disorder during the perinatal period and child outcomes: A systematic review. J Affect Disord. 2018 Jan 1;225:18-31.

9. Ehlers A, Clark DM. A cognitive model of posttraumatic stress disorder. Behav Res Ther. 2000;38(4):319-45.

10. Slade P, Atherton C, Kingdon C, Weeks A, Lavender T, Treadwell M, et al. Developing a package of care to identify and intervene with women at risk from post traumatic stress symptoms related to childbirth. Rep to Liverpool Heal Inequalities Fund, Liverpool Clin Comm Gr. 2014.

11. Gollwitzer PM, Sheeran P. Implementation intentions and goal achievement: A metaanalysis of effects and processes. Adv Exp Soc Psychol Vol 38. 2006;38:69-119.

12. Wessely S, Bryant RA, Greenberg N, Earnshaw M, Sharpley J, Hughes JH. Does Psychoeducation Help Prevent Post Traumatic Psychological Distress? Psychiatry Interpers Biol Process. 2008 Dec 16;71(4):287-302.

13. Turpin G, Downs M, Mason S. Effectiveness of providing self-help information following acute traumatic injury: Randomised controlled trial. Br J Psychiatry. 2005 Jul 2;187(01):76-82.

14. National Institute for Health and Care Excellence. Antenatal and postnatal mental health: clinical management and service guidance [CG192]. NICE; 2014 .

15. Devilly GJ, Gullo MJ, Alcorn KL, O’Donovan A. Subjective Appraisal of Threat (Criterion A2) as a Predictor of Distress in Childbearing Women. J Nerv Ment Dis. 2014 
Dec;202(12):877-82.

16. Zigmond AS, Snaith RP. The hospital anxiety and depression scale. Acta Psychiatr Scand . 1983 Jun;67(6):361-70.

17. Condon JT, Corkindale CJ. The assessment of parent-to-infant attachment: Development of a self-report questionnaire. J Reprod Infant Psychol. 1998;16(1):57.

18. Sabourin S, Valois P, Lussier Y. Development and Validation of a Brief Version of the Dyadic Adjustment Scale With a Nonparametric Item Analysis Model. Psychol Assess. 2005;17(1):15-27.

19. Charles JM, Edwards RT, Bywater T, Hutchings J. Micro-Costing in Public Health Economics: Steps Towards a Standardized Framework, Using the Incredible Years Toddler Parenting Program as a Worked Example. Prev Sci. 2013;14(4):377-89.

20. Curtis LA, Burns A. Unit costs of health and social care 2017. University of Kent; 2017. Available from: https://kar.kent.ac.uk/65559/

21. NHS Improvement. Reference costs 2016/17. 2017. Available from: https://improvement.nhs.uk/resources/reference-costs/

22. Braun V, Clarke V. Using thematic analysis in psychology. Qual Res Psychol. 2006 Jan;3(2):77-101.

23. Fleiss JL. Statistical methods for rates and proportions. 2nd edition. New York: John Wiley; 1981. 38-46 p.

24. Turpin G, Downs M, Mason S. Effectiveness of providing self-help information following acute traumatic injury: Randomised controlled trial. Br J Psychiatry. 2005;187(JULY):7682.

25. Neuner F, Schauer M, Klaschik C, Karunakara U, Elbert T. A Comparison of Narrative Exposure Therapy, Supportive Counseling, and Psychoeducation for Treating Posttraumatic Stress Disorder in an African Refugee Settlement. 2004; Available from:

http://citeseerx.ist.psu.edu/viewdoc/download?doi=10.1.1.467.1839\&rep=rep1\&type=pdf

26. Bryant RA, Harvey AG, Dang ST, Sackville T, Basten C. Treatment of acute stress disorder: A comparison of cognitive-behavioral therapy and supportive counseling. $\mathrm{J}$ Consult Clin Psychol. 1998;66(5):862-6.

27. Bryant RA, Sackville T, Dang ST, Moulds M, Guthrie R. Treating acute stress disorder: an evaluation of cognitive behavior therapy and supportive counseling techniques. Am $\mathrm{J}$ Psychiatry . $1999 ; 156(11): 1780-6$. 
28. Bryant RA, Moulds ML, Nixon R V.D. Cognitive behaviour therapy of acute stress disorder: a four-year follow-up. Behav Res Ther. 2003 Apr;41(4):489-94.

29. Ehlers A, Mayou R., Bryant B. Cognitive predictors of posttraumatic stress disorder in children: results of a prospective longitudinal study. Behav Res Ther. 2003 Jan;41(1):1-10.

30. Donker T, Griffiths KM, Cuijpers P, Christensen H. Psychoeducation for depression, anxiety and psychological distress: a meta-analysis. BMC Med. 2009 Dec 16;7(1):79.

31. Kawakami N, Haratani T, Iwata N, Imanaka Y, Murata K, Araki S. Effects of mailed advice on stress reduction among employees in Japan: A randomized controlled trial. Ind Health. 1999;37(2):237-42. 
Table 1: Demographic and Obstetric: Self-help (intervention) versus Usual care (control) (randomised women).

\begin{tabular}{|c|c|c|c|}
\hline \multicolumn{2}{|l|}{ Variable } & \multirow{2}{*}{$\begin{array}{l}\text { Self-help } p^{12} \\
\qquad N=346\end{array}$} & \multirow{2}{*}{$\begin{array}{c}\text { Usual care }{ }^{12} \\
\qquad \mathrm{~N}=345\end{array}$} \\
\hline & & & \\
\hline Age & mean(st. dev) & $30.10(5.09)$ & $30.39(5.26)$ \\
\hline Parity & median(IQR) & $1(1)$ & $1(1)$ \\
\hline & range & $1-5$ & $1-7$ \\
\hline \multirow[t]{4}{*}{ Ethnicity n(\%) } & White & $290(88.4)$ & 300 (90.6) \\
\hline & Asian/Asian British & $15(4.6)$ & $17(5.1)$ \\
\hline & Black/African/Caribbean & $3(0.9)$ & $3(0.9)$ \\
\hline & Other & $20(6.0)$ & $11(3.3)$ \\
\hline Days postnatal at & median(IQR) & $16(14)$ & $16(13)$ \\
\hline recruitment & Range & $2-70$ & $5-84$ \\
\hline Highest qualification $\mathrm{n}(\%)$ & Degree/Higher degree & $158(48.2)$ & $153(45.8)$ \\
\hline \multirow[t]{3}{*}{ Relationship status n(\%) } & Living together & $123(37.5)$ & $140(42.2)$ \\
\hline & Married & $168(51.2)$ & $160(48.2)$ \\
\hline & Single/divorced/widowed/not answered & $37(9.8)$ & $32(9.6)$ \\
\hline \multirow[t]{2}{*}{ Conception $\mathrm{n}(\%)$} & Natural & 339 (99.1) & 324 (95.9) \\
\hline & Assisted & $3(0.9)$ & $14(4.1)$ \\
\hline \multirow[t]{5}{*}{ Analgesia $\mathrm{n}(\%)$} & Regional anaesthetic & $166(48.0)$ & $173(51.2)$ \\
\hline & General anaesthetic & $20(5.8)$ & $24(7.2)$ \\
\hline & Inhaled nitrous oxide / oxygen & $59(17.1)$ & $62(18.6)$ \\
\hline & Opiates & 69 (19.9) & $52(15.6)$ \\
\hline & None /non pharm /not recorded & $32(9.2)$ & $22(6.6)$ \\
\hline \multirow[t]{6}{*}{ Place of birth $n(\%)$} & Theatre & $132(38.2)$ & 128 (37.9) \\
\hline & Midwife led unit & $55(15.9)$ & $47(13.9)$ \\
\hline & Consultant led unit & $154(44.5)$ & 155 (45.9) \\
\hline & Homebirth & $2(0.6)$ & $5(1.5)$ \\
\hline & Unplanned outside maternity unit & $2(0.6)$ & $3(0.9)$ \\
\hline & Maternity assessment unit & $1(0.3)$ & $0(0)$ \\
\hline Mode of birth $n(\%)$ & Spontaneous & $145(42.3)$ & $146(43.3)$ \\
\hline
\end{tabular}

This article is protected by copyright. All rights reserved 


\begin{tabular}{lrcc}
\hline & Instrumental & $85(24.8)$ & $71(21.1)$ \\
& Emergency CS & $91(26.5)$ & $84(24.9)$ \\
& Elective CS & $22(6.4)$ & $36(10.7)$ \\
\hline Labour induced $\mathrm{n}(\%)$ & $183(53.2)$ & $146(43.3)$ \\
\hline Episiotomy $\mathrm{n}(\%)$ & $82(23.1)$ & $74(21.9)$ \\
\hline Perineal trauma $\mathrm{n}(\%)$ & No & $239(70.9)$ & $230(70.3)$ \\
& $1^{\text {st }}$ degree perineal tear & $16(4.7)$ & $14(4.3)$ \\
& $2^{\text {nd }}$ degree perineal tear & $94(19.0)$ & $67(20.5)$ \\
\hline Blood loss $>\mathbf{1 0 0 0 m l} \mathrm{n}(\%)$ & $3^{\text {rd }}$ degree perineal tear & $18(5.3)$ & $13(4.3)$ \\
\hline Apgar $<7$ at $\mathbf{5}$ minutes $\mathrm{n}(\%)$ & $68(19.7)$ & $51(15.1)$ \\
\hline NICU admission $\mathrm{n}(\%)$ & $16(4.6)$ & $19(5.7)$ \\
\hline
\end{tabular}

${ }^{1}$ Includes women randomised in error.

${ }^{2}$ Numbers may not add up to total due to missing data. 
Table 2: Trial outcomes: Self-help (intervention) versus Usual care (control) Intention to treat analysis (followed-up women).

\begin{tabular}{|c|c|c|c|c|c|}
\hline \multirow[t]{2}{*}{ Variable } & & \multirow{2}{*}{$\begin{array}{l}\text { Self-help }{ }^{12} \\
N=243\end{array}$} & \multirow{2}{*}{$\begin{array}{c}\text { Usual care }{ }^{12} \\
\qquad \mathrm{~N}=244\end{array}$} & \multirow{2}{*}{$\begin{array}{l}\text { Difference }(95 \% \mathrm{Cl}) \\
\text { Relative risk }(95 \% \mathrm{Cl})\end{array}$} & \multirow[t]{2}{*}{ Significance } \\
\hline & & & & & \\
\hline \multirow[t]{3}{*}{ PTSD Diagnosis } & None & $178(73.3)$ & $179(73.7)$ & & \\
\hline & Partial & $49(20.2)$ & $43(17.7)$ & & \\
\hline & Full & $16(6.6)$ & $21(8.6)$ & $1.02(0.68,1.53)^{6,7}$ & $P=0.92^{3}$ \\
\hline CAPS Criterion A met n(\%) & No & $23(9.5)$ & $18(7.4)$ & & \\
\hline (Trauma exposure) & Yes & $220(90.5)$ & $226(92.6)$ & $0.87(0.61,1.24)^{6}$ & $P=0.41^{3}$ \\
\hline CAPS Criterion B met n(\%) & No & $151(63.4)$ & $142(59.2)$ & & \\
\hline (Intrusion symptoms) & Yes & $87(36.6)$ & $98(40.18)$ & $0.92(0.76,1.10)^{6}$ & $P=0.34^{3}$ \\
\hline CAPS Criterion C met n(\%) & No & $197(81.1)$ & $181(74.5)$ & & \\
\hline (Avoidance symptoms) & Yes & $46(18.9)$ & $62(25.5)$ & $0.83(0.67,1.01)^{6}$ & $P=0.08^{3}$ \\
\hline CAPS Criterion D met n(\%) & No & $181(74.8)$ & $178(73.3)$ & & \\
\hline (Cognitions \& mood symptoms) & Yes & $61(25.2)$ & $65(26.7)$ & $0.96(0.79,1.17)^{6}$ & $P=0.70^{3}$ \\
\hline CAPS Criterion E met $n(\%)$ & No & $208(86.0)$ & $203(83.5)$ & & \\
\hline (Arousal \& reactivity symptoms) & Yes & $34(14.0)$ & $40(16.5)$ & $0.91(0.73,1.15)^{6}$ & $P=0.46^{3}$ \\
\hline CAPS Criterion G met n(\%) & No & $169(69.8)$ & $168(68.1)$ & & \\
\hline (Distress \& impairment symptoms) & Yes & $73(30.2)$ & 75 (30.9) & $0.98(0.81,1.19)^{6}$ & $P=0.87^{3}$ \\
\hline HADS Anxiety mean(st. dev) & & $5.63(4.16)$ & $5.40(3.95)$ & $-0.23(-0.97,0.49)^{5}$ & $P=0.53^{4}$ \\
\hline
\end{tabular}

This article is protected by copyright. All rights reserved 


\begin{tabular}{lcccc}
\hline HADS Depression mean(st. dev) & $3.77(3.23)$ & $4.13(3.43)$ & $0.35(-0.28,0.91)^{5}$ & $P=0.25^{4}$ \\
\hline MPAS Quality of attachment mean(st. dev) & $41.02(4.92)$ & $40.57(4.97)$ & $-0.43(-1.31,0.45)^{5}$ & $P=0.34^{4}$ \\
\hline MPAS Absence of hostility mean(st. dev) & $20.87(3.68)$ & $20.52(3.32)$ & $-0.33(-0.98,0.28)^{5}$ & $P=0.28^{4}$ \\
\hline MPAS Pleasure in interaction mean(st. dev) & $22.27(3.35)$ & $22.39(3.81)$ & $0.10(-0.54,0.74)^{5}$ & $P=0.76^{4}$ \\
\hline DAS4 total mean(st. dev) & $17.13(3.61)$ & $17.00(3.63)$ & $-0.12(-0.76 .0 .52)^{5}$ & $P=0.71^{4}$
\end{tabular}

${ }^{1}$ Includes women randomised in error.

${ }^{2}$ Numbers may not add up to total due to missing data.

${ }^{3}$ Chi-squared test

${ }^{4}$ Independent sample t-test

${ }^{5}$ Mean difference

${ }^{6}$ Relative risk

${ }^{7}$ Comparison full/partial against none 
Table 3: Trial outcomes: Self-help (intervention) versus Usual care (control) Intention to treat analysis adjusted for induction and Blood loss $>1000 \mathrm{ml}$ (followed-up women).

\begin{tabular}{|c|c|c|c|c|c|}
\hline \multirow[t]{2}{*}{ Variable } & & Self-help ${ }^{12}$ & Usual care $^{12}$ & Adjusted Odds & Significance \\
\hline & & $N=243$ & $N=244$ & ratio $(95 \% \mathrm{Cl})$ & \\
\hline \multirow[t]{2}{*}{ PTSD Diagnosis } & None & $178(73.3)$ & $179(73.7)$ & & \\
\hline & Partial/Full & $65(26.7)$ & $63(26.63$ & $0.99(0.65,1.49)$ & $P=0.95^{3}$ \\
\hline CAPS Criterion A met $n(\%)$ & No & $23(9.5)$ & $18(7.4)$ & & \\
\hline (Trauma exposure) & Yes & $220(90.5)$ & $226(92.6)$ & $0.70(0.35,1.35)$ & $P=0.28^{3}$ \\
\hline CAPS Criterion B met n(\%) & No & $151(63.4)$ & $142(59.2)$ & & \\
\hline (Intrusion symptoms) & Yes & $87(36.6)$ & $98(40.18)$ & $0.82(0.56,1.19)$ & $P=0.29^{3}$ \\
\hline CAPS Criterion C met n(\%) & No & $197(81.1)$ & $181(74.5)$ & & \\
\hline (Avoidance symptoms) & Yes & $46(18.9)$ & $62(25.5)$ & $0.64(0.41,0.99)$ & $P=0.047^{3}$ \\
\hline CAPS Criterion D met $n(\%)$ & No & $181(74.8)$ & $178(73.3)$ & & \\
\hline (Cognitions \& mood symptoms) & Yes & $61(25.2)$ & $65(26.7)$ & $0.86(0.57,1.31)$ & $P=0.71^{3}$ \\
\hline CAPS Criterion E met $n(\%)$ & No & $208(86.0)$ & $203(83.5)$ & & \\
\hline (Arousal \& reactivity symptoms) & Yes & $34(14.0)$ & $40(16.5)$ & $0.72(0.43,1.21)$ & $P=0.21^{3}$ \\
\hline CAPS Criterion G met $n(\%)$ & No & $169(69.8)$ & $168(68.1)$ & & \\
\hline (Distress \& impairment symptoms) & Yes & $73(30.2)$ & $75(30.9)$ & $0.92(0.62,1.38)^{6}$ & $P=0.69^{3}$ \\
\hline HADS Anxiety mean(st. dev) & & $5.63(4.16)$ & $5.40(3.95)$ & $-0.23(-0.97,0.49)^{5}$ & $\mathrm{P}=0.97^{4}$ \\
\hline
\end{tabular}

This article is protected by copyright. All rights reserved 
HADS Depression mean(st. dev)

${ }^{1}$ Includes women randomised in error

${ }^{2}$ Numbers may not add up to total due to missing data.

${ }^{3}$ Logistic regression

${ }^{4}$ Analysis of covariance

${ }^{5}$ Mean difference

${ }^{6}$ Relative risk

This article is protected by copyright. All rights reserved 
2 Figure 1: CONSORT diagram

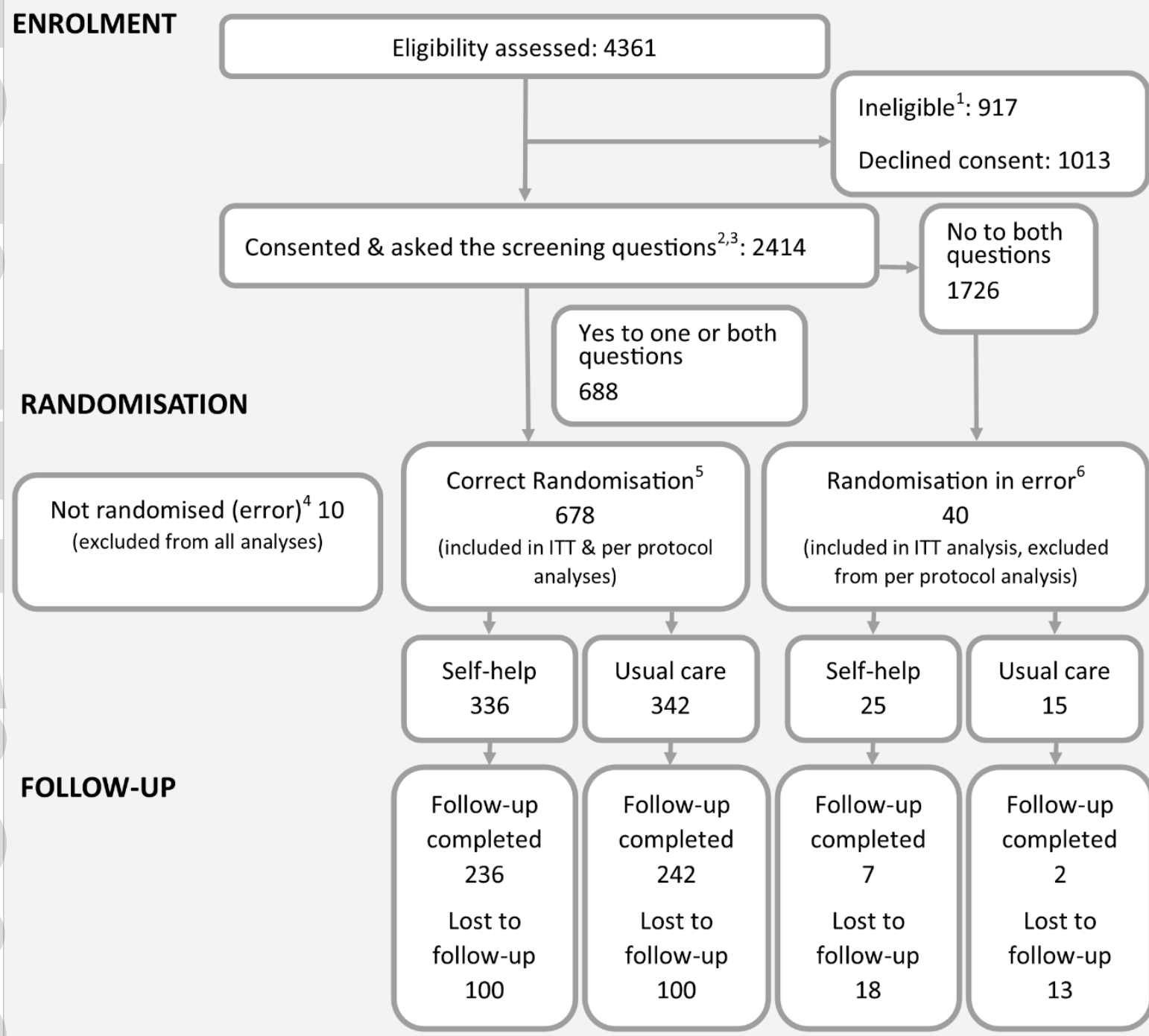

Due to the large number of individuals recruiting to the study (125 community midwives), randomisation errors sometimes occurred:

${ }^{1}$ Seven women consented and were randomised in error despite being ineligible (did not speak sufficient English to complete follow-up interview, birth was outside the Trust, or mother was receiving specialist support).

${ }^{2}$ An additional fifteen women were randomised, but no documents were returned to the research office, or the consent form was returned unsigned.

${ }^{3}$ Two women screened negative, but no documents were returned to the research office, or the consent form was returned unsigned.

${ }^{4}$ Ten women answered 'yes' to one or both questions, but were not randomised.

${ }^{5}$ Eight women were randomised twice. Where group was unclear, analysis was according to the allocation of the second randomisation.

${ }^{6}$ Forty women were randomised in error having answered 'no' to both screening questions. 